\title{
Real-World Efficacy of Fulvestrant Monotherapy as the First Treatment or Maintenance Treatment in Patients with Metastatic Breast Cancer
}

\author{
Meng Lv Yan Mao Teng Ma Yongmei Wang Xiaoyi Liu Yuhua Song \\ Haibo Wang
}

Breast Disease Center, Affiliated Hospital of Qingdao University, Qingdao, PR China

\section{Keywords}

Metastatic breast cancer · Fulvestrant monotherapy · Real world $\cdot$ Maintenance treatment $\cdot$ First-line treatment

\begin{abstract}
Background: Fulvestrant 500 mg monotherapy is recommended as the first-line endocrine treatment in postmenopausal women with hormone receptor-positive metastatic breast cancer (MBC). It is also used in MBC maintenance treatment. However, few studies have compared the efficacy of fulvestrant during the initial treatment with that during maintenance treatment. Patients and Methods: MBC patients who were treated with fulvestrant either as initial therapy for metastatic disease or after progression following one line of chemotherapy between January 2016 and December 2017 were identified from the database of the Affiliated Hospital of Qingdao University. The primary end point was progression-free survival (PFS). Results: The study included 135 MBC patients who were treated with fulvestrant; 116 patients who received fulvestrant as first-line treatment were divided into 2 groups: the no-chemotherapy treatment (NCT) group received fulvestrant as initial therapy during disease progression, and the chemotherapy treatment (CT) group received fulvestrant as maintenance following disease stabilization or response to previous chemotherapy. The median PFS was 16 months in NCT patients and 8 months in the CT group. Patients who had a longer disease-free survival, no visceral metastasis and one metastasis site, benefited from fulvestrant as first-line treatment during disease progression. Patients with 2 or more metastasis sites bene-
\end{abstract}

fited from chemotherapy as first-line treatment and fulvestrant as maintenance treatment. Conclusions: Fulvestrant monotherapy showed good clinical activity and safety in patients with $\mathrm{MBC}$ who were treated upon disease progression and in those receiving maintenance therapy.

(c) 2020 The Author(s)

Published by S. Karger AG, Basel

\section{Introduction}

Breast cancer is a common malignant tumor in women worldwide. The incidence of breast cancer in the United States accounts for $30 \%$ of the total cancer incidence, and the mortality rate is approximately $15 \%$ according to the American Cancer Society. In China, the incidence and mortality of breast cancer in women are 15 and $6 \%$, respectively $[1,2]$. Hormone receptor (HR)positive breast cancer accounts for $70-80 \%$ of all breast cancers. Approximately $40 \%$ of women who present with early-stage HR-positive breast cancer eventually develop metastatic disease [3]. Among these metastatic patients, endocrine therapy (ET) is the first choice of treatment as either the first line or second line even if patients have visceral metastasis (but not in cases of visceral crisis or ET resistance) [4]. The choice of endocrine drug depends on the previous treatment regimen and disease-free survival (DFS), and includes tamoxifen, aromatase inhibitors (AIs), or fulvestrant. However, some patients who are initially sensitive to ET develop resistance to ET [5]. Hence, the strategy and sequence of ET drugs are important.
() 2020 The Author(s)

Published by S. Karger AG, Basel

This is an Open Access article licensed under the Creative Commons Attribution-NonCommercial-4.0 International License (CC BY-NC) (http://www.karger.com/Services/OpenAccessLicense), applicable to the online version of the article only. Usage and distribution for commercial purposes requires written permission.
Meng Lv and Haibo Wang

Breast Disease Center, Affiliated Hospital of Qingdao University

Qingdao, Haier Road No. 59

Qingdao, Shandong 266000 (PR China)

lvmengstudy @163.com and hbwang66@126.com 
Table 1. Clinical characteristics of ER-positive patients with fulvestrant usage $(n=135)$

\begin{tabular}{|c|c|c|c|c|}
\hline \multirow[t]{2}{*}{ Covariate level } & \multirow{2}{*}{$\begin{array}{l}\text { All patients } \\
(n=135)\end{array}$} & \multicolumn{2}{|c|}{ Fulvestrant usage } & \multirow[t]{2}{*}{$p$ value } \\
\hline & & $\begin{array}{l}\text { first line } \\
(n=116)\end{array}$ & $\begin{array}{l}\geq \text { second line } \\
(n=19)\end{array}$ & \\
\hline Age at diagnosis, years & $53(27-82)$ & $53(27-82)$ & $52(30-71)$ & 0.792 \\
\hline $\begin{array}{l}\text { Age at fulvestrant usage, median } \\
\text { years }\end{array}$ & $57(30-67)$ & $57(30-67)$ & $55(35-52)$ & 0.620 \\
\hline \multicolumn{5}{|l|}{$\mathrm{ER}, n(\%)$} \\
\hline Negative & $14(10.37)$ & $12(10.34)$ & $2(10.5)$ & \multirow{2}{*}{0.977} \\
\hline Positive & $121(89.63)$ & $104(89.65)$ & $17(89.5)$ & \\
\hline \multicolumn{5}{|l|}{$\mathrm{PR}, n(\%)$} \\
\hline Negative & $27(20.00)$ & $22(18.97)$ & $5(26.32)$ & \multirow[t]{2}{*}{0.438} \\
\hline Positive & $108(80.00)$ & $94(81.03)$ & $14(73.68)$ & \\
\hline \multicolumn{5}{|l|}{ HER2, $n(\%)$} \\
\hline Negative & $108(80.00)$ & $91(78.44)$ & $17(86.47)$ & \multirow[t]{3}{*}{0.538} \\
\hline Positive & $14(10.37)$ & $13(11.20)$ & $1(5.26)$ & \\
\hline Unknown & $13(9.63)$ & $12(9.48)$ & $1(5.26)$ & \\
\hline \multicolumn{5}{|l|}{ Histological grade, $n(\%)$} \\
\hline $\mathrm{I}$ & $15(11.11)$ & $12(10.34)$ & $3(15.79)$ & \multirow[t]{3}{*}{0.080} \\
\hline II & $68(50.37)$ & $56(48.28)$ & $12(63.16)$ & \\
\hline III & $52(38.52)$ & $48(41.38)$ & $4(21.05)$ & \\
\hline \multicolumn{5}{|l|}{ Stage, $n(\%)$} \\
\hline $\mathrm{I}$ & $21(15.55)$ & $20(17.44)$ & $1(5.26)$ & \multirow[t]{4}{*}{0.205} \\
\hline II & $49(36.30)$ & $39(33.85)$ & $10(52.63)$ & \\
\hline III & $53(39.26)$ & 45 (38.79) & $8(42.11)$ & \\
\hline IV & $12(8.89)$ & $12(10.34)$ & $0(0)$ & \\
\hline \multicolumn{5}{|l|}{ Metastatic sites, $n(\%)$} \\
\hline Lymph nodes & $43(31.85)$ & $40(34.88)$ & $3(15.79)$ & \multirow[t]{3}{*}{0.364} \\
\hline Bone & $80(59.26)$ & $68(58.62)$ & $12(63.12)$ & \\
\hline Visceral & $69(51.11)$ & $62(53.44)$ & $7(36.84)$ & \\
\hline
\end{tabular}

Fulvestrant, a selective estrogen receptor (ER) degrader, binds to the ER, downregulating its expression and blocking ER function. It binds with 100-fold greater affinity than tamoxifen, and it is more effective than tamoxifen and AIs in inhibiting estrogen signaling [6, 7]. The CONFIRM trial, a phase 3 double-blind trial, divided patients with metastatic breast cancer (MBC) that progressed after previous endocrine treatment with tamoxifen or AIs into 2 groups: those receiving fulvestrant $500 \mathrm{mg}$ and those receiving $250 \mathrm{mg}$ [8]. The study showed that fulvestrant at $500 \mathrm{mg}$ significantly improved progression-free survival (PFS) and the overall response rate compared with fulvestrant $250 \mathrm{mg}$. Hence, in subsequent clinical trials or in the clinic, fulvestrant $500 \mathrm{mg}$ is the first choice compared with fulvestrant $250 \mathrm{mg}$. The First trial, a phase 2, double-blind clinical trial, randomized women who had not been previously treated into 2 groups treated with fulvestrant $500 \mathrm{mg}$ or anastrozole [9]. The fulvestrant $500 \mathrm{mg}$ group showed significantly improved PFS and overall survival compared with the anastrozole group. These results were confirmed in the phase 3 FALCON trial. The median PFS was 16.6 months in the fulvestrant $500 \mathrm{mg}$ group and 13.8 months in the anastrozole group [10]. The American Society of Clinical Oncology therefore recommends fulvestrant 500 $\mathrm{mg}$ as the first-line treatment in postmenopausal women with HR-positive MBC.

However, breast cancer patients in clinical trials and in real world practice are different. For example, patients in the First and FALCON trials did not receive chemotherapy. In clinical practice, some patients receive chemotherapy before fulvestrant after progression. There are few data on the efficacy and safety of fulvestrant treatment in realworld practice, and whether fulvestrant is more effective as first-line or maintenance therapy remains unclear. In this study, we collected real-world data to retrospectively analyze the patterns of treatment and efficacy of fulvestrant in $\mathrm{MBC}$ patients, and we analyzed the factors affecting the response to treatment in HR-positive MBC patients.

\section{Materials and Methods}

Study Population

MBC patients who were treated with fulvestrant between January 2016 and December 2017 were included for study from the database of the Affiliated Hospital of Qingdao University. All tumors were ER and/or progesterone receptor (PR) positive (defined as ER or PR $>1 \%$ ) as determined by immunohistochemistry. All patients received fulvestrant $500 \mathrm{mg}$ every 28 days after progres- 
sion. Clinical data collected included age, ER/PR/HER2 status, histological grade, TNM stage, metastatic sites, and prior endocrine and cytotoxic chemotherapies.

\section{Treatment Plan}

HR-positive MBC patients received fulvestrant $500 \mathrm{mg}$ until progressive disease, unacceptable toxicity, or loss of follow-up according to the approved national guidelines. Efficacy was determined according to Response Evaluation Criteria in Solid Tumors (RECIST) 1.1 criteria.

\section{Statistical Analysis}

The primary end point of this study was PFS, defined as the time from initiation of post fulvestrant treatment to disease progression. The secondary aim of this study was the evaluation of adverse events, which were determined retrospectively based on medical records, including leukopenia, thrombocytopenia, anemia, hand-foot syndrome, atrial fibrillation, increased alanine aminotransferase levels, oral mucositis, myalgia, and nausea. The Kaplan-Meier and log-rank methods were used to analyze PFS curves. Differences were assessed using the $\chi^{2}$ and Kruskal-Wallis tests. The risk factors and adjusted hazard ratios with $95 \%$ confidence intervals were calculated using the multivariable Cox proportional hazards model. All statistical analyses were performed using SPSS (version 23.0). Data were considered significant at $p<$ 0.05 .

\section{Results}

\section{Patients and Treatment}

In total, 135 patients with MBC from the Affiliated Hospital of Qingdao University breast cancer database treated with fulvestrant $500 \mathrm{mg}$ between January 2016 and December 2017 were analyzed. The clinical features of these patients are presented in Table 1.

The study included 135 patients, of which $116(85.9 \%)$ received fulvestrant as first-line ET and 19 (14.1\%) received fulvestrant as the second line or later lines of ET. There were no significant differences in clinical features such as age, tumor grade, ER/PR/HER2 expression levels, stage, and metastatic sites (Table 1). However, our study indicated that patients with later stage and worse grade would prefer to receive fulvestrant as the second line.

Nineteen patients received other endocrine treatments before fulvestrant including NSAIDs (nonsteroidal antiinflammatory drugs), exemestane, or exemestane + everolimus. To identify the factors affecting the efficacy of first-line fulvestrant treatment, we divided the $116 \mathrm{pa}-$ tients who received fulvestrant as the first-line ET into 2 groups, a no-chemotherapy treatment (NCT) group and a chemotherapy treatment (CT) group before fulvestrant treatment during disease progression. The most frequently $(\geq 10 \%)$ used chemotherapy regimens were docetaxel-based in $42.5 \%$, capecitabine in $27.5 \%$, and carboplatin-based in $20 \%$ of patients.

Of these patients, 76 (65.5\%) did not receive chemotherapy before fulvestrant, whereas 40 (34.5\%) were treat-
Table 2. Clinical characteristics of NCT and CT group patients

\begin{tabular}{|c|c|c|c|}
\hline Characteristics & $\begin{array}{l}\text { NCT, \% } \\
(76 ; 65.5 \%)\end{array}$ & $\begin{array}{l}\text { CT, \% } \\
(40 ; 34.5 \%)\end{array}$ & $\begin{array}{l}p \\
\text { value }\end{array}$ \\
\hline Median age, years & 58 & 54 & 0.604 \\
\hline DFI, $n(\%)$ & & & 0.603 \\
\hline$\leq 24$ months & $23(30.3)$ & $14(35.0)$ & \\
\hline$>24$ months & $53(69.7)$ & $26(65.0)$ & \\
\hline $\mathrm{ER}, n(\%)$ & & & 0.701 \\
\hline Negative & $6(7.9)$ & $4(10.0)$ & \\
\hline Positive & $70(92.1)$ & $36(90.0)$ & \\
\hline $\mathrm{PR}, n(\%)$ & & & 0.344 \\
\hline Negative & $17(22.4)$ & $6(15.0)$ & \\
\hline Positive & $59(77.6)$ & $34(85.0)$ & \\
\hline HER2, $n(\%)$ & & & 0.112 \\
\hline Negative & $62(81.6)$ & $31(77.5)$ & \\
\hline Positive & $5(6.58)$ & $7(17.5)$ & \\
\hline Unknown & $9(11.8)$ & $2(5.0)$ & \\
\hline Histological grade, $n(\%)$ & & & 0.359 \\
\hline I & $4(5.3)$ & $3(7.5)$ & \\
\hline II & $41(53.9)$ & $26(65.0)$ & \\
\hline III & $31(40.8)$ & $11(27.5)$ & \\
\hline Stage, $n(\%)$ & & & 0.041 \\
\hline $\mathrm{I}$ & $16(21.5)$ & $2(5.0)$ & \\
\hline II & $30(32.9)$ & $13(32.5)$ & \\
\hline III & $28(40.8)$ & $17(42.5)$ & \\
\hline IV & $2(3.9)$ & $8(5.0)$ & \\
\hline Metastatic sites, $n(\%)$ & & & 0.312 \\
\hline Lymph nodes & $11(14.5)$ & $12(30.0)$ & \\
\hline Bone & $39(51.3)$ & $23(57.5)$ & \\
\hline Visceral & $35(46.1)$ & $18(45.0)$ & \\
\hline $\begin{array}{l}\text { Number of metastatic sites, } n \\
(\%)\end{array}$ & & & 0.022 \\
\hline 1 & $56(73.7)$ & $21(52.5)$ & \\
\hline$\geq 2$ & $20(26.3)$ & $19(47.5)$ & \\
\hline \multicolumn{4}{|l|}{ DFS, months, $n(\%)$} \\
\hline$\leq 24$ & $23(30.3)$ & $14(35.0)$ & 0.677 \\
\hline$>24$ & $53(69.7)$ & $26(65.0)$ & \\
\hline
\end{tabular}

DFI, disease-free interval.

ed with chemotherapy. The baseline characteristics of patients are summarized in Table 2 . The median age was 58 years in the NCT group and 54 years in the CT group. The highest DFS period was $>24$ months in both NCT and CT groups. There were no statistically significant differences in HR/HER2 status, histological grade, tumor stage, and metastatic sites (lymph nodes, bone, and visceral metastatic sites). However, there were more patients with 2 or more metastatic sites in the CT group than in the NCT group (47.5 and $26.3 \%$, respectively).

\section{Clinical Outcomes and Safety}

The median PFS for all patients was 10 months (Fig. 1A). Patients receiving first-line fulvestrant had better metastatic PFS (mPFS) than those receiving secondline fulvestrant (12 vs. 6 months; $p=0.000$; Fig. 1B). NCT patients had better PFS than CT patients (16 vs. 8 months; 


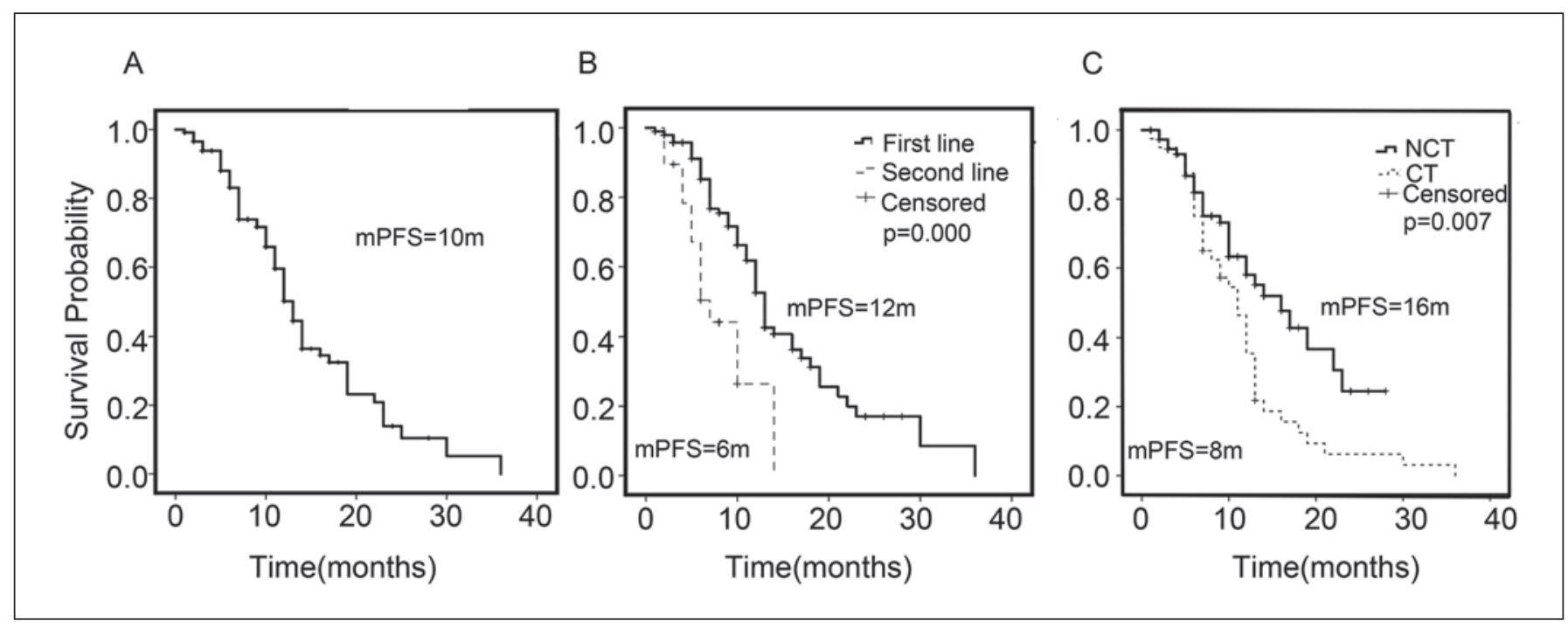

Fig. 1. Kaplan-Meier curves for progression-free survival probabilities for all enrolled 116 patients (A), stratified by fulvestrant lines $(\mathbf{B})$ and previous treatment $(\mathbf{C})$.

$p=0.007$; Fig. 1C). Subgroup analysis showed that among patients with a shorter DFS ( $\leq 24$ months), NCT patients had a significantly longer PFS than CT patients $(p=0.009$; Fig. 2A). However, in patients with a longer DFS ( $>24$ months), the PFS was similar between the NCT and CT groups ( $p=0.110$; Fig. $2 \mathrm{~B}$ ).

PFS was significantly longer in the NCT group than in the CT group among patients without visceral metastasis $(p=0.008$; Fig. 2D). However, there were no significant differences in PFS between the 2 groups among patients with bone metastasis ( $p=0.085$; Fig. $2 \mathrm{C}$ ). Among patients with one metastasis site, the PFS was longer in NCT patients than in CT patients ( $p=0.025$; Fig. $2 \mathrm{E}$ ). In patients with 2 or more metastasis sites, the mPFS of the CT group was longer than that of the NCT group (Fig. 2F), although the difference did not reach statistical significance $(p=$ $0.135)$.

In the multivariate Cox model, the choice of chemotherapy before fulvestrant treatment was an important factor for PFS (Table 3). The results indicated that in patients with a longer DFS, without visceral metastasis, or one metastasis site, omitting chemotherapy before fulvestrant treatment was a beneficial factor.

\section{Treatment Safety}

The grade $3 / 4$ adverse events are summarized in Table 4. Hematological toxicities and nausea were significantly more frequent in the CT group than in the NCT group (12.5 vs. $1.3 \%$ and 15 vs. $1.3 \%$, respectively; $p<0.05$ ). Hand-foot syndrome, atrial fibrillation, and increased alanine aminotransferase were only observed in the CT group (7.5 vs. $0 \%$ ). Oral mucositis and myalgia, arthralgia, and hot flushes occurred in both groups.

\section{Discussion}

HR-positive breast cancer is the most common subtype in both young and old women. ET is the mainstay of treatment for HR-positive postmenopausal advanced breast cancer patients. Compared with AI, fulvestrant is currently the preferred standard first-line therapy for HR-positive postmenopausal women with MBC.

Similarly, in our study, 116 (85.9\%) patients received fulvestrant as first-line ET, and 19 (14.1\%) patients received fulvestrant as the second line or later lines of ET. Even though there were no significant differences in clinical features, there was a little difference. Our study showed that patients with later stage and worse grade received fulvestrant as the second-line treatment. We think reasons may be as follows: first, most doctors think that patients with later stage and worse grade should firstly receive chemotherapy. The maintenance treatment with AI or NSAI drugs are sufficient after chemotherapy. Second, the price of fulvestrant is higher than that of AI or NSAI. Some patients chose fulvestrant as the second-line treatment. Third, the sample is small, especially the patients who received fulvestrant as the second-line treatment. There was some selection bias.

In daily clinical practice, switching to ET during or after chemotherapy is a common strategy that is used for maintaining efficacy and decreasing the incidence of side effects. Such a strategy is also supported by the European Consensus Guidelines with a level of evidence of IC (class I/C level) [11]. In China, physicians recommend first-line chemotherapy for younger patients with late stage disease and 2 or more metastatic sites. This was also reported by Dufresne et al. [12], who showed that patients with lymph node-positive disease, multiple metastatic sites, and a lon- 
Fig. 2. Kaplan-Meier curves for progression-free survival probabilities stratified by previous treatment in patients with different disease-free survival (A, B), bone metastasis $(\mathbf{C})$, without visceral metastasis (D), with 1 metastasis site (E), and with 2 or more metastasis sites $(\mathbf{F})$. NCT, metastatic breast cancer patients during disease progression did not receive chemotherapy treatment; CT, metastatic breast cancer patients received chemotherapy treatment before fulvestrant treatment during disease progression.

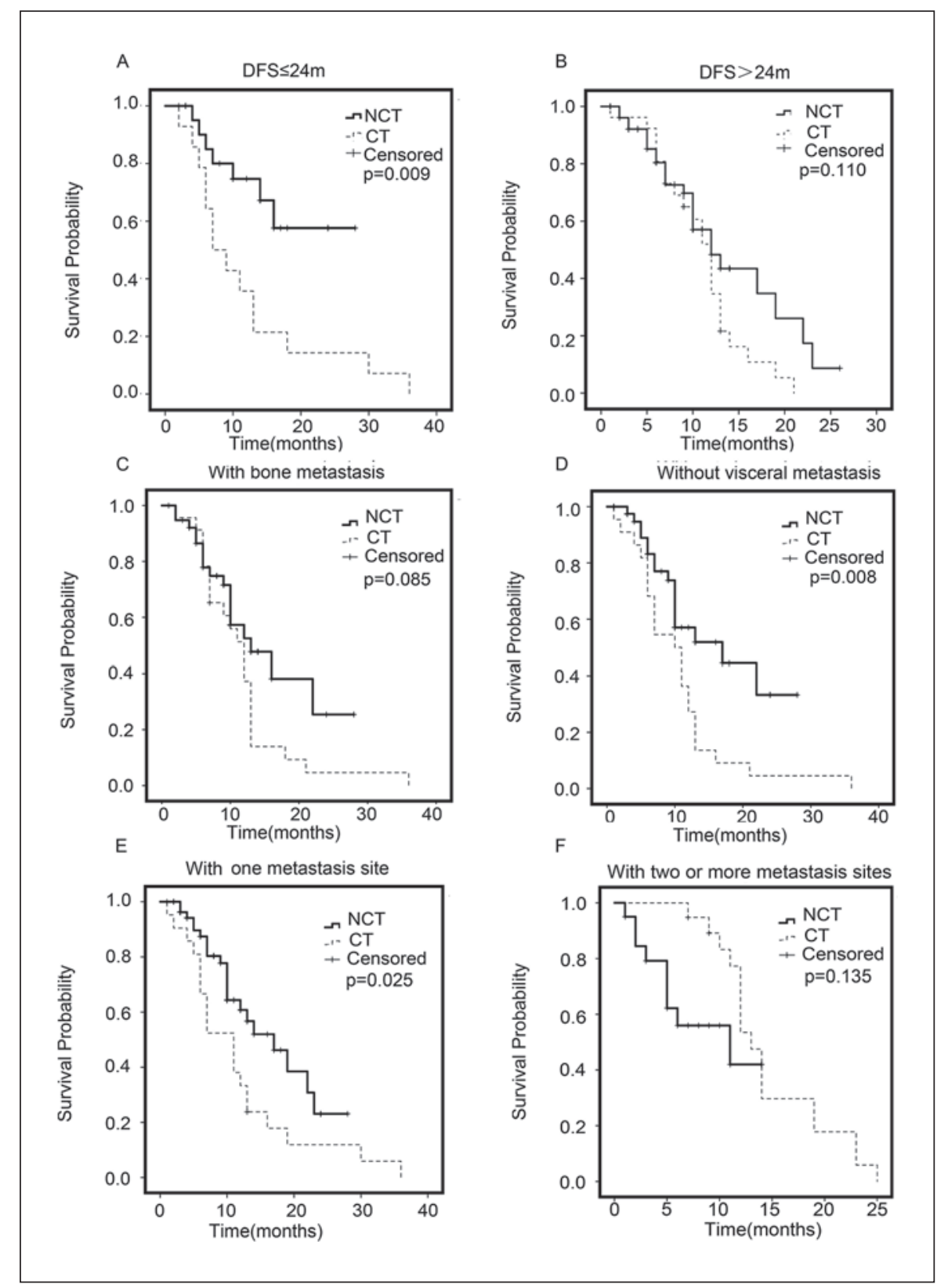

ger disease-free interval usually receive chemotherapy as the first-line treatment and ET as the maintenance treatment. Because the risk of recurrence is higher in these patients, they should receive chemotherapy firstly to prevent recurrence or metastasis progression. In addition, doctors tend to directly choose ET for patients with a lower tumor burden and a better prognosis and for patients with boneonly metastasis [13]. Lobbezoo et al. [14] performed a retrospective analysis and reported that bone-only metastasis should be an indication for an ET-first approach. Nikura et al. [15] demonstrated that ET is not inferior to chemotherapy in HR+/HER2- MBC patients with boneonly metastasis. Consistent with these findings, the CT group in this study included a greater number of patients with a higher tumor burden (later stage and 2 metastasis sites). Our study showed that most Chinese doctors prefer to choose chemotherapy as first treatment for patients with a higher tumor burden and then switching to ET.

In this study, the mPFS for all users, first-line users, and second users was 10,12, and 6 months, respectively. The mPFS for first-line users in our study was shorter than the mPFS reported in clinical trials. The FALCON trial showed that fulvestrant significantly improved the PFS (mPFS, 16.6 months) for patients with HR-positive locally advanced or metastatic breast cancer who did not receive previous ET [10]. The mPFS in patients receiving second-line fulvestrant in this study was similar to that reported in clinical trials. The CONFIRM phase 3 trial enrolled 735 HR-positive advanced breast cancer patients who experienced progression after prior ET from 17 coun- 
Table 3. Cox proportional hazard analysis of influence on progression-free survival

\begin{tabular}{|c|c|c|c|}
\hline Variables & Hazard ratio & $95 \%$ CI & $p$ value \\
\hline \multicolumn{4}{|l|}{ Age } \\
\hline$<60$ years & reference & & \\
\hline$\geq 60$ years & 0.990 & $0.423-2.371$ & 0.982 \\
\hline \multicolumn{4}{|l|}{ Tumor grade } \\
\hline I & reference & & \\
\hline II & 1.700 & $0.474-6.097$ & 0.415 \\
\hline III & 2.179 & $0.798-5.000$ & 0.066 \\
\hline \multicolumn{4}{|l|}{ Tumor stage } \\
\hline I & reference & & \\
\hline II & 1.660 & $0.162-17.013$ & 0.669 \\
\hline III & 1.045 & $0.110-9.903$ & 0.969 \\
\hline IV & 0.763 & $0.082-7.094$ & 0.812 \\
\hline \multicolumn{4}{|l|}{ ER status } \\
\hline Negative & reference & & \\
\hline Positive & 0.372 & $0.066-2.095$ & 0.262 \\
\hline \multicolumn{4}{|l|}{ PR status } \\
\hline Negative & reference & & \\
\hline Positive & 1.252 & $0.472-3.322$ & 0.652 \\
\hline \multicolumn{4}{|l|}{ HER2 status } \\
\hline Unknown & reference & & \\
\hline Negative & 1.384 & $0.190-10.087$ & 0.749 \\
\hline Positive & 1.270 & $0.449-3.588$ & 0.593 \\
\hline \multicolumn{4}{|l|}{ DFS, months } \\
\hline$\leq 24$ & reference & & \\
\hline$>24$ & 0.488 & $0.209-1.142$ & 0.098 \\
\hline \multicolumn{4}{|c|}{ Metastatic sites } \\
\hline 1 & reference & & \\
\hline$\geq 2$ & 1.123 & $0.432-4.321$ & 0.538 \\
\hline \multicolumn{4}{|c|}{ Bone metastasis } \\
\hline No & reference & & \\
\hline Yes & 1.244 & $0.455-3.403$ & 0.670 \\
\hline \multicolumn{4}{|c|}{ Visceral metastasis } \\
\hline No & reference & & \\
\hline Yes & 1.454 & $0.494-4.279$ & 0.497 \\
\hline \multicolumn{4}{|l|}{ CT before } \\
\hline No & reference & & \\
\hline Yes & 2.225 & $1.097-4.510$ & 0.027 \\
\hline
\end{tabular}

tries. The results showed that fulvestrant $500 \mathrm{mg}$ improved the clinical benefit in MBC patients previously treated with ET. The mPFS was 6.5 months in this trial [8]. This could be attributed to the fact that most patients in the FALCON trial did not receive any treatment including neoadjuvant and adjuvant treatment. In this study, most patients received adjuvant ET, and ET may not be effective in these patients. The present study and clinical trials suggested that the early administration of fulvestrant was associated with better patient prognosis. In addition, after multiline ET, the ER status may change, which may lead to the development of drug resistance. Similarly, our study suggested that HR-positive MBC patients should receive fulvestrant treatment as early as possible.

Several clinical studies demonstrated the efficacy and safety of fulvestrant treatment and proposed that fulves-
Table 4. Treatment-related adverse events

\begin{tabular}{lcl}
\hline Adverse event (grade 3/4) & $\begin{array}{l}\text { NCT }(n=76), \\
n(\%)\end{array}$ & $\begin{array}{l}\text { CT }(n=40), \\
n(\%)\end{array}$ \\
\hline Leukopenia & $0(0)$ & $4(10)$ \\
Trombocytopenia & $0(0)$ & 0 \\
Anemia & $1(1.3)$ & $1(2.5)$ \\
Hand-foot syndrome & $0(0)$ & $1(2.5)$ \\
Atrial fibrillation & $0(0)$ & $1(2.5)$ \\
Alanine aminotransferase increased & $0(0)$ & $1(2.5)$ \\
Oral mucositis & $2(2.6)$ & $1(2.5)$ \\
Myalgia, arthralgia & $6(7.9)$ & $1(5)$ \\
Hot flushes & $23(30.2)$ & $11(27.5)$ \\
Nausea & $1(1.3)$ & $6(15)$ \\
\hline
\end{tabular}

trant should be the first-line therapy for $\mathrm{MBC}$ patients because these patients have a relatively good prognosis compared with those receiving multiline therapy $[16,17]$. In these trials, the rate of patients who received chemotherapy was low. In the FALCON trial, one third of the patients had previously received CT. However, in clinical practice in China, HR-positive MBC patients usually first receive chemotherapy before receiving ET. Dufresne et al. [12] examined the efficacy of chemotherapy (capecitabine) compared with hormonal therapy after response to firstline chemotherapy in patients with HR-positive and HER2-negative breast cancer. They found that hormonal therapy can improve the time to progress over chemotherapy ( 13 vs. 8 months; $p=0.011$ ). In addition, patients in the hormonal therapy group had fewer adverse events, whereas patients in the MCT group experienced grade 3-4 adverse events [18]. Another study showed that patients with HR+ HER2- MBC receiving maintenance hormonal therapy had a long PFS and overall survival after first-line chemotherapy (16.3 and 48.8 months, respectively) [12].

Whether HR-positive MBC patients should directly receive fulvestrant treatment or receive maintenance fulvestrant treatment after chemotherapy for first-line ET remains unclear. An Italian clinical study showed that patients with de novo metastatic disease have a relatively good prognosis compared with those with recurring disease. However, there was no difference in MPFS between patients receiving fulvestrant treatment at progressive disease or those receiving fulvestrant as maintenance after chemotherapy (11.6 and 11.1 months, respectively) [19]. This result was slightly different from those of the present study. The present study examined the efficacy of prefulvestrant treatment in ER+/HER2- MBC patients in China. The results showed that the NCT group had a longer PFS than the CT group. This can be explained as follows: first, many patients had 2 and more metastatic sites in the CT group. The prognosis of these patients is usually worse. Second, there were few patients with de novo metastatic 
disease in our study, and most of them received adjuvant chemotherapy or adjuvant endocrine therapy. Our result indicated that the prognosis was better for patients firstly receiving fulvestrant treatment at progressive disease than those receiving fulvestrant as maintenance after chemotherapy. In other words, the earlier fulvestrant is used, the better prognosis patients would have.

Bone metastasis is the most common site in HR-positive breast cancer patients [20]. A recent analysis confirmed that breast cancer patients with bone-only metastasis have a better PFS and a good response to ET [21]. However, bone-only disease is not as common, and it is often studied in combination with other visceral sites or lymph nodes in clinical practice. In this study, there were few patients with bone-only metastasis, and most patients had bone metastasis together with visceral sites or lymph node metastasis. We found no differences in the NCT group and CT group between those with or without bone metastasis. One reason for this result could be that most patients have a combination of bone and non-bone metastatic sites. The prognosis of these patients is poor, and they show a poor response to ET. However, patients without visceral metastases are often better likely to respond to ET than those with visceral metastases. In the FALCON study [10], the mPFS was longer in patients without visceral disease than in those with visceral disease (22.3 and 13.8 months, respectively) after first-line fulvestrant treatment $[10,11]$. Similarly, in this study, the mPFS was longer in the NCT group than in the CT group in patients without visceral metastases. However, in patients with visceral metastases, the mPFS of the NCT group was slightly longer than that of the CT group (although not statistically significant). These findings indicated that for patients with visceral metastases and 2 or more metastatic sites, fulvestrant was suitable as the maintenance treatment after chemotherapy. However, for patients without visceral metastases, the prognosis was better to receive fulvestrant treatment after disease progression.

The present study had several limitations. First, this study had a retrospective design. The critical issue addressed was which patients should receive fulvestrant directly and which ones should be treated with maintenance fulvestrant. This question remained unanswered because we did not perform a clinical trial. Second, there was some selection bias because data were derived from a single institution and the number of patients was too small to draw firm conclusions.

\section{Conclusion}

The earlier fulvestrant is used for advanced breast cancer patients, the better prognosis patients would have. Fulvestrant first-line users had a median PFS of 12 months which was longer than that of the second-line users (6 months). Most Chinese doctors prefer to choose chemotherapy as first treatment for patients with a higher tumor burden and switching to ET. In addition, patients with a shorter DFS, 1 metastasis site and without visceral metastasis should receive fulvestrant treatment as soon as possible after disease progression. However, patients with 2 or more metastasis sites could receive chemotherapy as first treatment and switch to ET.

\section{Acknowledgment}

The authors thank the patients and family members for their participation in this study.

\section{Statement of Ethics}

The study protocol has been approved by the Affiliated Hospital of Qingdao University on human research. All subjects have given their written informed consent.

\section{Disclosure Statement}

We have no conflicts of interest to disclose.

\section{Funding Sources}

The study was supported by the Natural Science Foundation of China (No. 81772845), Natural Science Doctoral Fundings of Shandong province (No. ZR2019BH013, ZR2017BH061, ZR2018BH023) and by the grants from Clinical Medicine + X Project of the Affiliated Hospital of Qingdao University (No. 3424).

\section{Author Contributions}

M.L.: data analysis, interpretation of data, drafting article. T.M.: data collection. Y.M.: interpretation of data and writing assistance. Y.W.: data collection and data analysis. X.L.: literature search. Y.S.: study concept and design. H.W.: publication decision.

\section{References}

1 Siegel RL, Miller KD, Jemal A. Cancer statistics, 2019. CA Cancer J Clin. 2019 Jan;69(1):7-34.

2 Chen W, Zheng R, Baade PD, Zhang S, Zeng H, Bray F, et al. Cancer statistics in China, 2015. CA Cancer J Clin. 2016 Mar-Apr;66(2):115-32.

3 Park S, Han W, Kim J, Kim MK, Lee E, Yoo TK, et al. Risk factors associated with distant metastasis and survival outcomes in breast cancer patients with locoregional recurrence. J Breast Cancer. 2015 Jun; 18(2):160-6.

4 Gradishar WJ, Anderson BO, Balassanian R, Blair SL, Burstein HJ, Cyr A, et al. Breast Cancer, Version 4.2017, NCCN Clinical Practice Guidelines in Oncology. J Natl Compr Canc Netw. 2018 Mar;16(3):310-20. 
5 Augereau P, Patsouris A, Bourbouloux E, Gourmelon C, Abadie Lacourtoisie S, Berton Rigaud D, et al. Hormonoresistance in advanced breast cancer: a new revolution in endocrine therapy. Ther Adv Med Oncol. 2017 May;9(5):335-46.

6 Howell A, Robertson J F R, Quaresma Albano J, Aschermannova A, Mauriac L, Kleeberg UR, et al. Fulvestrant, formerly ICI 182,780, is as effective as anastrozole in postmenopausal women with advanced breast cancer progressing after prior endocrine treatment. J Clin Oncol. 2002 Aug;20(16):3396-403.

7 Howell A, Robertson JF, Abram P, Lichinitser MR, Elledge R, Bajetta E, et al. Comparison of fulvestrant versus tamoxifen for the treatment of advanced breast cancer in postmenopausal women previously untreated with endocrine therapy: a multinational, double-blind, randomized trial. J Clin Oncol. 2004 May;22(9): 1605-13.

8 Di Leo A, Jerusalem G, Petruzelka L, Torres $\mathrm{R}$, Bondarenko IN, Khasanov R, et al. Results of the CONFIRM phase III trial comparing fulvestrant $250 \mathrm{mg}$ with fulvestrant $500 \mathrm{mg}$ in postmenopausal women with estrogen receptor-positive advanced breast cancer. J Clin Oncol. 2010 Oct;28(30):4594-600.

9 Ellis MJ, Llombart-Cussac A, Feltl D, Dewar JA, Jasiówka M, Hewson N, et al. Fulvestrant $500 \mathrm{mg}$ versus anastrozole $1 \mathrm{mg}$ for the firstline treatment of advanced breast cancer: overall survival analysis from the phase II FIRST study. J Clin Oncol. 2015 Nov;33(32): 3781-7.

10 Robertson JF, Bondarenko IM, Trishkina E Dvorkin M, Panasci L, Manikhas A, et al. Fulvestrant $500 \mathrm{mg}$ versus anastrozole $1 \mathrm{mg}$ for hormone receptor-positive advanced breast cancer (FALCON): an international, randomised, double-blind, phase 3 trial. Lancet. 2016 Dec;388(10063):2997-3005.
11 Cardoso F, Costa A, Senkus E, Aapro M, André $\mathrm{F}$, Barrios $\mathrm{CH}$, et al. 3rd ESO-ESMO international consensus guidelines for Advanced Breast Cancer (ABC 3). Ann Oncol. 2017 Jan;28(1):16-33.

12 Dufresne A, Pivot X, Tournigand C, Facchini $\mathrm{T}$, Alweeg T, Chaigneau L, et al. Maintenance hormonal treatment improves progression free survival after a first line chemotherapy in patients with metastatic breast cancer. Int J Med Sci. 2008 May;5(2):100-5.

13 Watanabe J, Hayashi T, Tadokoro Y, Nishimura S, Takahashi K. Clinical pattern of primary systemic therapy and outcomes of estrogen receptor-positive, HER2-negative metastatic breast cancer: a review of a single institution. Breast Cancer Res Treat. 2017 Dec;166(3):911-7.

14 Lobbezoo DJ, van Kampen RJ, Voogd AC, Dercksen MW, van den Berkmortel F, Smilde $\mathrm{TJ}$, et al. In real life, one-quarter of patients with hormone receptor-positive metastatic breast cancer receive chemotherapy as initial palliative therapy: a study of the Southeast Netherlands Breast Cancer Consortium. Ann Oncol. 2016 Feb;27(2):256-62.

15 Niikura N, Liu J, Hayashi N, Palla SL, Tokuda Y, Hortobagyi GN, et al. Treatment outcome and prognostic factors for patients with boneonly metastases of breast cancer: a single-institution retrospective analysis. Oncologist. 2011;16(2):155-64.

16 Dawood S, Broglio K, Ensor J, Hortobagyi GN, Giordano SH. Survival differences among women with de novo stage IV and relapsed breast cancer. Ann Oncol. 2010 Nov; 21(11):2169-74
17 Lobbezoo DJ, van Kampen RJ, Voogd AC, Dercksen MW, van den Berkmortel F, Smilde TJ, et al. Prognosis of metastatic breast cancer: are there differences between patients with de novo and recurrent metastatic breast cancer? Br J Cancer. 2015 Apr;112(9):1445-51.

18 Chen XL, Du F, Hong RX, Wang JY, Luo Y, Li $\mathrm{Q}$, et al. Hormonal therapy might be a better choice as maintenance treatment than capecitabine after response to frst-line capecitabine-based combination chemotherapy for patients with hormone receptor-positive and HER2-negative, metastatic breast cancer. Chin J Cancer. 2016;25:35-9.

19 Palumbo R, Sottotetti F, Quaquarini E, Gambaro A, Ferzi A, Tagliaferri B, et al. Patterns of treatment and outcome with 500-mg fulvestrant in postmenopausal women with hormone receptor-positive/HER2-negative metastatic breast cancer: a real-life multicenter Italian experience. Ther Adv Med Oncol. 2019 Jun;11:1758835919833864.

20 Coleman RE. Clinical features of metastatic bone disease and risk of skeletal morbidity. Clin Cancer Res. 2006 Oct;12(20 Pt 2):6243s9s.

21 Wedam SB, Beaver JA, Amiri-Kordestani L, Bloomquist E, Tang S, Goldberg KB, et al. US Food and Drug Administration Pooled Analysis to Assess the Impact of Bone-Only Metastatic Breast Cancer on Clinical Trial Outcomes and Radiographic Assessments. J Clin Oncol. 2018 Apr;36(12):1225-31.

22 Turner NC, Finn RS, Martin M, Im SA, DeMichele A, Ettl J, et al. Clinical considerations of the role of palbociclib in the management of advanced breast cancer patients with and without visceral metastases. Ann Oncol. 2018 Mar;29(3):669-80. 